\title{
Data processing and analyzing of magnetotellurics survey data in time domain
}

\author{
Hikaru NAGATA ${ }^{1}$, Hitoshi MIKADA ${ }^{1}$, Tada-nori GOTO ${ }^{1}$, \\ Junichi TAKEKAWA ${ }^{1}$ and Takafumi KASAYA ${ }^{2}$ \\ ${ }^{1}$ Dept. of Civil and Earth Res. Eng., Kyoto University \\ ${ }^{2}$ JAMSTEC.
}

\begin{abstract}
Data processing of magnetotelluric (MT) survey has been based on the Fast Fourier Transform (FFT). The FFT processing gives us the response functions (RFs) of the earth fast and easily. However, applying FFT processing to MT data may not be optimum always, since the source of MT is the transient fluctuation of electric current in the ionosphere. The FFT assumes time series to be a stationary so that we focused on an IIR filter called 'pole on pedestal' that extracts the signal at a specific frequency. Combining this IIR filter and the Hilbert transform, the RFs are calculated in time domain. For removing the segment contaminated by noise before calculating the RFs, we applied the maximum entropy method (MEM) to the selection of the segments contaminated by large noise. The MEM searches and removes these contaminated segments easily. As a result, we developed the time domain processing of MT data using MEM and IIR filter, and applied this processing to the real data acquired at the Nankai trough. Comparing the conventional and novel data processing, our novel data processing gives us more optimum RFs than the conventional processing. In addition, we discussed the time-domain separation of up-going/down-going electromagnetic waves based on the plane wave decomposition. All our attempts support an idea that the time-domain analysis of magnetotelluric data would give us more accurate and detailed subsurface information.
\end{abstract}

\section{Introduction}

Data processing is one of the essential techniques to obtain optimum response functions (RFs) of the earth. The processing of magnetotelluric (MT) survey data is based on the spectrum analysis using the Fast Fourier Transform (FFT). The robust processing based on FFT has been used widely ${ }^{1)}$. This processing gives us optimum RFs of the earth when the $\mathrm{S} / \mathrm{N}$ ratio is high and the time window length is long enough. However, the errors of response function become very large when the data quality is low or the length of time-series is short. This reason is that MT data is in general non-stationary since the source of MT is the transient electromagnetic fluctuation in the ionosphere. On the other hand, FFT assumes input time series to be stationary. Therefore, when the duration of input signal is short, we do not get optimum response function since the stationary assumption of FFT does not satisfy. In order to solve this problem, we developed the data processing without FFT by using a IIR filter. In addition, it is very important for the data processing of MT to select the analyzed segments or remove the segments contaminated by noise. Robust and coherence sort algorithm have been developed and applied in order to select the segments contaminated by noise. However, these methods are sometimes insufficient to remove the contaminated segments. We developed the segments selection algorithm by using MEM. We apply our novel data processing to the real data acquired at Nankai trough and compared to the conventional method.

\section{METHOD}

We focus on an IIR filter to calculate the RFs in time domain without FFT. This IIR filter is called 'pole on pedestal' 2) that extracts and amplifies signals at a specific frequency. Pole on pedestal defines $Z$ transform (Eq.1).

$$
\mathrm{H}(\mathrm{z})=\frac{\mathrm{SZ}}{p Z_{0}-Z} \cdot \frac{\mathrm{SZ}}{\bar{Z}_{0}-\bar{Z}}
$$

There is the opposite relation between pole on pedestal filter and notch filter. Eq.1 is defined by multiplying two transfer functions whose relation is time reversal, so that we can make zero phase filter. $\mathrm{S}$ and $\mathrm{q}$ are the locations of pole and zero point on the $\mathrm{Z}$ plane.

\subsection{CALCULATE RFs IN TIME DOMAIN}


Applying pole on pedestal filter, we can get the signal at a specific frequency. The extracted signal has the real part of the wave. We should calculate the imaginary part of the wave (complex wave) because the signal of MT survey data has the phase. In order to calculate the imaginary part, we applied the Hilbert transform to the real part. Combining the real and imaginary part, complex wave in time domain is calculated. The impedance is usually calculated by Eq.2. [AB] in Eq.2 is cross spectrum in frequency domain. In this study, it is complex cross correlation in time domain. We can calculate the RFs in time domain using pole on pedestal and Hilbert transform.

$$
Z x x=\frac{\left[E_{x} \bar{H}_{x}\right]\left[H_{y} \bar{H}_{y}\right]-\left[E_{x} \bar{H}_{y}\right]\left[H_{y} \bar{H}_{x}\right]}{\left[H_{x} \bar{H}_{x}\right]\left[H_{y} \bar{H}_{y}\right]-\left[H_{x} \bar{H}_{y}\right]\left[H_{y} \bar{H}_{x}\right]}
$$

In the MT processing, acquired data is usually divided into some segments. However, all segments are not analyzed since some segments are contaminated by noise. We must choose and analyze the relevant segments. Robust and coherence sort algorithm have been developed and applied. These methods are sometimes insufficient to remove the contaminated segments. Kimura et al. $(2005)^{3)}$ selects the segment by their eyes before using the coherence and robust algorithm. Calculated RFs is more accurate than only Robust and coherence algorithm. However, eyes selection is subjective, so we developed another algorithm. We use the maximum entropy method (MEM) to select the segments. We can get the power spectrum of each component (Ex or Hx etc.) by applying the MEM. We focus on the plane wave assumption of
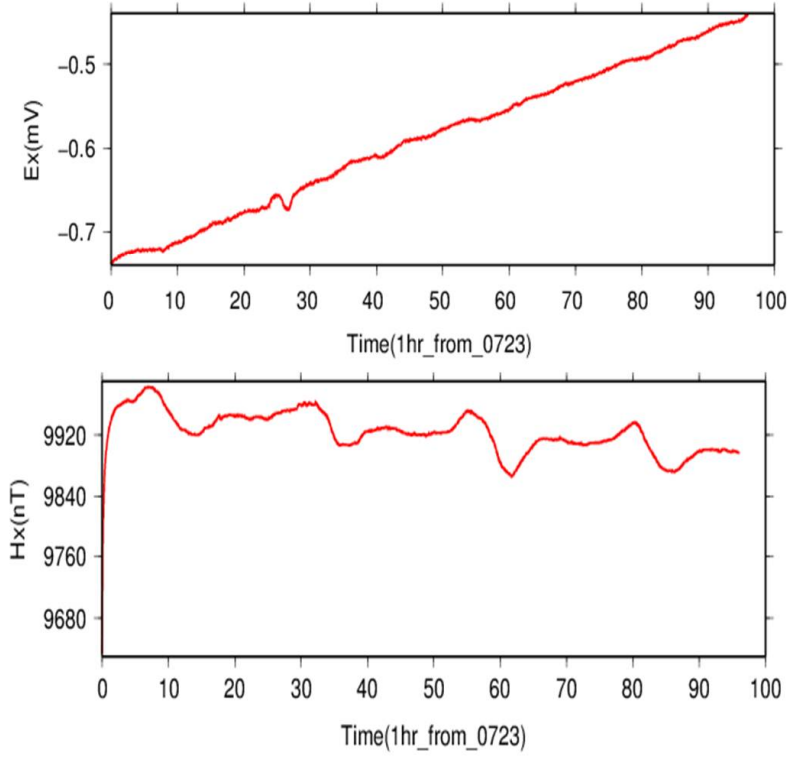

MT in order to search the contaminated segments. The same magnetic field signal penetrates at the local and remote (reference) sites, so the power spectrums of magnetic field between local and remote sites should be similar. When spectrums are compared between local and remote sites and there are some spectrum peaks at only local sites, we assume that these peaks were caused by noise and do not analyze the segment at the frequency.

\section{FIELD DATA ANALYSIS}

We apply the developed data processing to the real data acquired near Nankai trough. This data was recorded by ocean bottom electro-magnetic meter (OBEM). Fig.1 shows the raw data of each component. The strong geomagnetic variation did not occur during the observation term, so the signal was very weak and the duration of the signal was very short. We analyzed this data by developed and conventional processing. Fig. 2 shows the apparent resistivity of Zxy and Zyx. The red lines were calculated by time domain processing and the blue lines was calculated by frequency domain processing. We used the software 'RRRMT' to calculate the impedance by frequency domain processing. The error of the apparent resistivity calculated by time domain processing is much smaller than frequency domain processing. The error amplitudes calculated by frequency domain processing are between $40 \%$ and $100 \%$ (see Fig. 3). On the other hand, it calculated by time domain processing is below $40 \%$. We can get more optimum RFs of the earth by using time domain processing. We confirm that it is difficult to get optimum RFs from the data processing based on FFT when the duration of input signal is short and
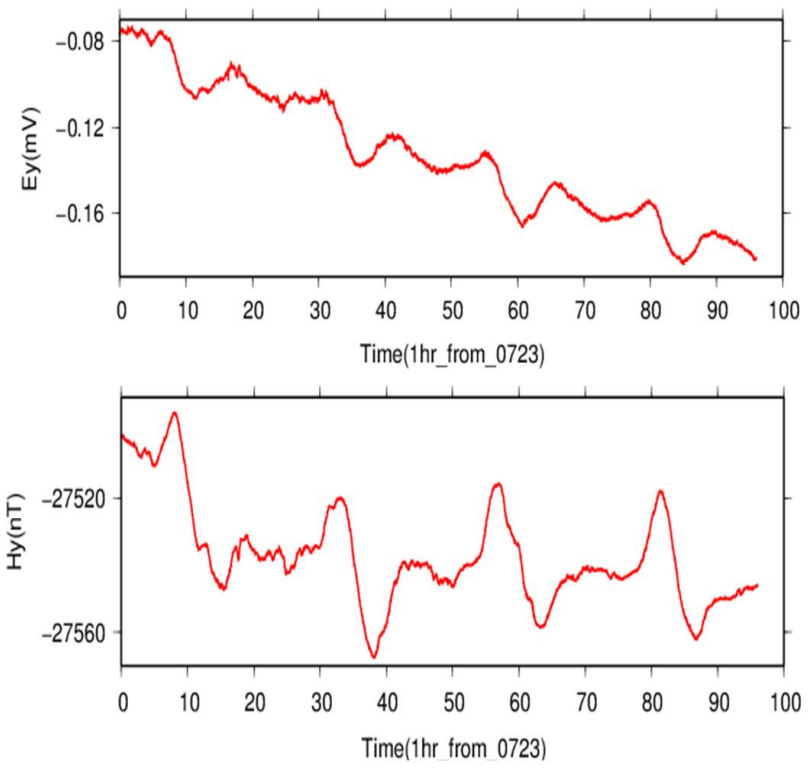

Figure 1 The raw data acquired at the Nankai trough 
$\mathrm{S} / \mathrm{N}$ ratio is low. The time domain processing by using IIR filter is efficient for analyzing the short and low $\mathrm{S} / \mathrm{N}$ data.
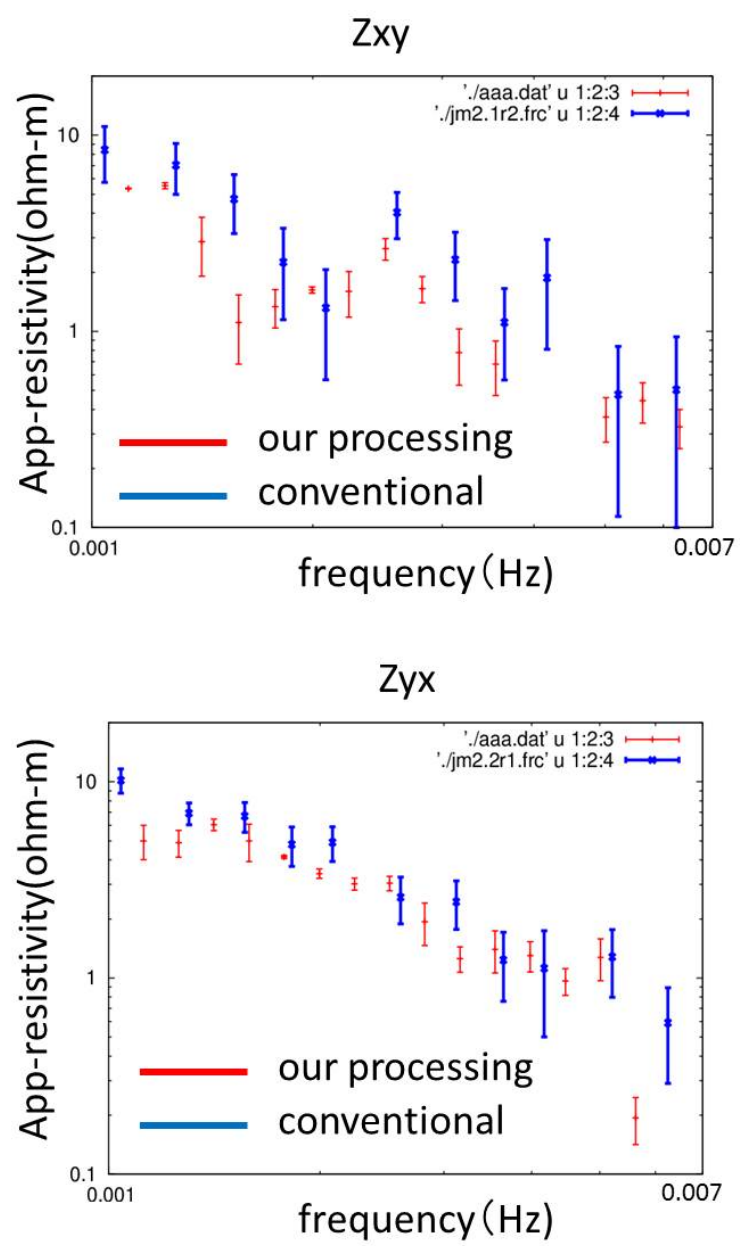

Figure 2 Apparent resistivity of Zxy and Zyx calculated by our processing (red) and conventional process (blue).

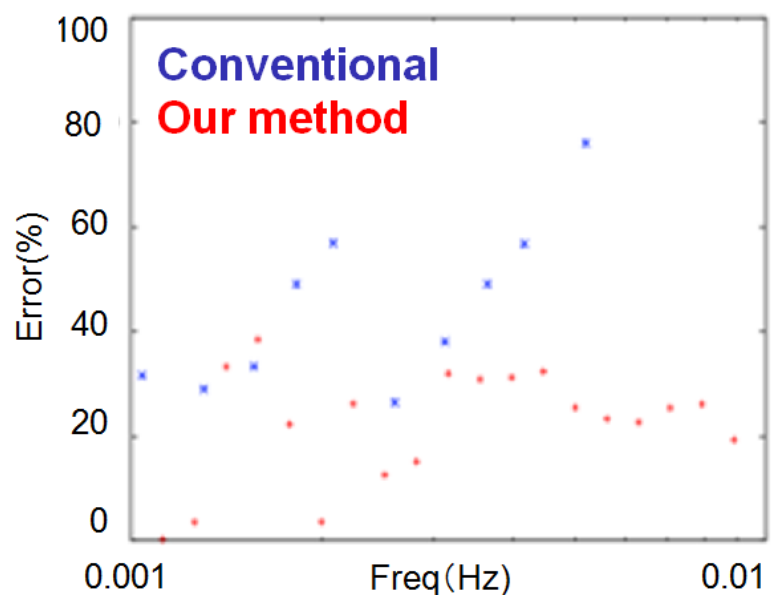

Figure 3 Errors of apparent resistivity of Zxy calculated by our processing (red) and conventional process (blue).

\section{PLANE WAVE DECOMPOSITION}

Although the magnetotelluric survey is a powerful tool for imaging the subsurface resistivity structure, time-domain electromagnetic data can give us further possibility of high-resolution imaging. For example, the migration procedure at seismic reflection survey, seismic interferometry and so on can be applied to the electromagnetic wave propagations for imaging the source locations (e.g., resistivity anomaly) under the ground or seafloor.

For testing the hypothesis, we compared the electric field obtained at the Nankai trough, as introduced above. We adopted our filtering method to the electric field of Ex component (N30W-S30E direction) to the marine magnetotelluric dataset obtained by three different OBEMs (Fig. 4). Although the water depths of three sites are similar, the amplitude and phase among three indicates the clear difference. It implies that the sub-seafloor structure may generate the variations of time series obtained by three OBEMs. In the other words, we have a possibility to obtain underground information from time-domain data analysis.

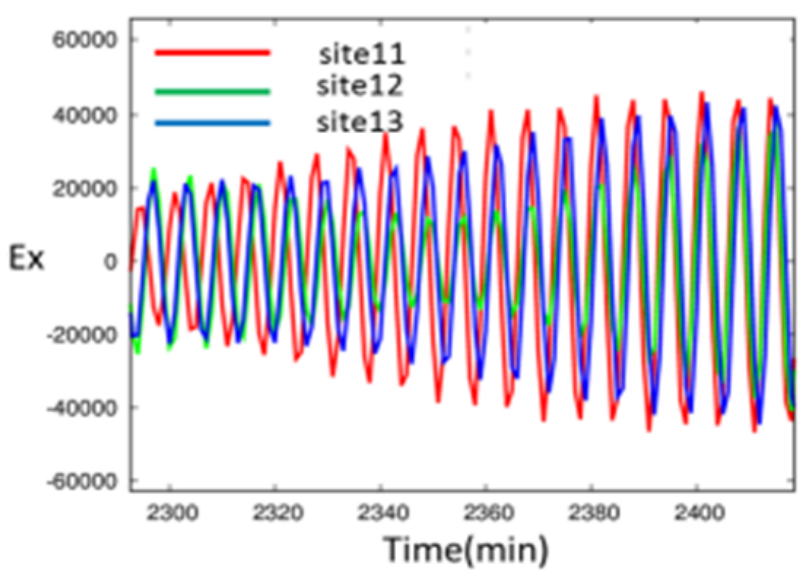

Figure 4 Filtered time series data of Ex component from different OBEMs (sites 11-13).

Here, for demonstration of time-domain data analysis, the plane wave decomposition of electromagnetic field is tested numerically. The plane wave decomposition, which allows us to separate up-going and down-going electromagnetic waves, is based on a theory presented by Amundsen et al. (2006). The original theory is designed for the controlled-source electromagnetic survey, but can be used for magnetotelluric.

The electromagnetic field on the surface was numerically calculated by using three-dimensional finite difference time domain (FDTD) method. 
Fig. 5 indicates the simplified subsurface resistivity model. Two magnetotelluric sites are assumed on the surface and two-dimensional conductive zone is embedded below the surface. We deployed 30 magnetotelluric receivers on the surface, and two of them are plotted in Fig. 5. The distance between two sites (Rec1 \& Rec2) is $2 \mathrm{~km}$. The model size is $20 \mathrm{~km} \times 20 \mathrm{~km} \times 20 \mathrm{~km}$ with grid size of $500 \mathrm{~m}$.

The obtained up-going / down-going electrical fields were then estimated by using the plane wave decomposition. Fig. 5 indicates that amplitude and phase of electric field is strongly affected by the subsurface resistivity anomaly. Thus, the array observation of magnetotellurics, often carried out recently, and the time-domain data analysis give us chance to extract the subsurface information directly from the time series.

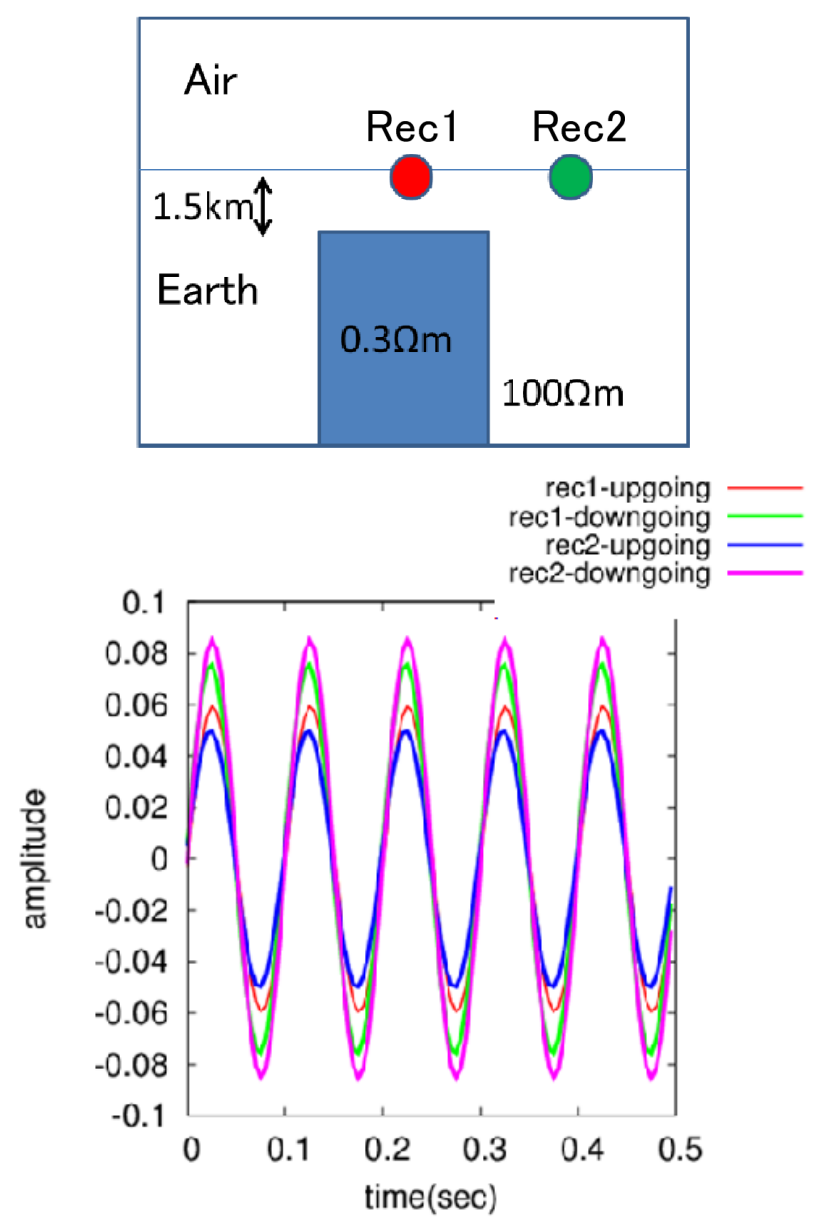

Figure 5 Numerical test of the plane wave decomposition. Upper: the model. Lower: decomposed electric field at receivers 1 and 2.

\section{CONCLUTION}

We developed the novel data processing in timedomain using the Hilbert transform and IIR filter called pole on pedestal which extracts and amplifies the signal at a specific frequency. In addition, we apply the MEM to select the segment contaminated by noise. We applied and compared developed time domain and conventional frequency domain processing to the real data acquired at Nankai trough. The length of the segments included in the signal is very short and signal power is very small. The RFs calculated by time domain processing are more accurate than the processing based on FFT. We conclude that time-domain processing using IIR filter and MEM gives us more optimum RFs when the length of data is short and $\mathrm{S} / \mathrm{N}$ ratio is low. In addition, we discussed the time-domain separation of up-going/down-going electromagnetic waves based on the plane wave decomposition. These results strongly suggest that the time-domain data analysis give us chance to extract the subsurface information directly from the time series, not from the magnetotelluric impedance tensors. It will result in higher resolution for imaging the resistivity structure due to the great increase of observed information.

\section{REFERENCES}

1) Chave, A.D. and Thomson, D. J. (1989): some comments on Magnetotelluric Response Function Estimation, Journal of Geophysics Research, 94, B10, 14, 215- 225.

2) Claerbout, J.F. (1976): Fundamentals of Geophysical Data Processing: With Applications to Petroleum Processing, McFraw-Hill

3) Kimura, T., Ashida, Y., Goto, T., Kasaya, T., Mikada, H., Sanada, Y., Watanabe, T. and Yamane, K.(2005): Crustal resistivity structure around the Nankai subduction zone: Geophysical Exploration (Butsuri-Tansa), 58, 251-262.

4) Amundsen, L., Loseth, L., Ellingsrud, S. and Ursin B. (2006): Decomposition of electromagnetic fields into upgoing and downgoing components, Geopphysics, 71, G211-G223. 